\title{
Elastomeric Tiles for the Fabrication of Inflatable Structures
}

\section{Citation}

Morin, Stephen A., Sen Wai Kwok, Joshua Lessing, Jason Ting, Robert F. Shepherd, Adam A. Stokes, and George M. Whitesides. 2014. "Elastomeric Tiles for the Fabrication of Inflatable Structures." Adv. Funct. Mater. 24 (35) (July 9): 5541-5549. doi:10.1002/adfm.201401339.

\section{Published Version}

doi:10.1002/adfm.201401339

\section{Permanent link}

http://nrs.harvard.edu/urn-3:HUL.InstRepos:16920699

\section{Terms of Use}

This article was downloaded from Harvard University's DASH repository, and is made available under the terms and conditions applicable to Open Access Policy Articles, as set forth at http:// nrs.harvard.edu/urn-3:HUL.InstRepos:dash.current.terms-of-use\#OAP

\section{Share Your Story}

The Harvard community has made this article openly available.

Please share how this access benefits you. Submit a story.

Accessibility 


\title{
Elastomeric Tiles for the Fabrication of Inflatable Structures
}

\author{
Stephen A. Morin ${ }^{1}$, Sen Wai Kwok ${ }^{1}$, Joshua Lessing ${ }^{1}$, Jason Ting ${ }^{1}$, Robert F. Shepherd ${ }^{1}$, Adam \\ A. Stokes ${ }^{1}$, and George M. Whitesides ${ }^{* 1,2,3}$ \\ Department of Chemistry and Chemical Biology, Harvard University \\ 12 Oxford Street, Cambridge, MA 02138
}

\footnotetext{
*Author to whom correspondence should be addressed

${ }^{1}$ Department of Chemistry and Chemical Biology

Harvard University

12 Oxford Street, Cambridge, MA 02138, USA

Email: gwhitesides@gmwgroup.harvard.edu

${ }^{2}$ Kavli Institute for Bionano Science \& Technology

Harvard University

29 Oxford Street, Cambridge, MA 02138, USA

${ }^{3}$ Wyss Institute for Biologically Inspired Engineering

Harvard University

60 Oxford Street, Cambridge, MA 02138, USA
}

Target journal: Advanced Functional Materials 


\section{Abstract}

This paper describes the fabrication of three-dimensional (3D) soft, inflatable structures from thin, two-dimensional (2D) tiles fabricated from elastomeric polymers. The tiles were connected using soft joints that increased the surface area available for gluing them together, and mechanically reinforced the structures to withstand the tensile forces associated with pneumatic actuation. The ability of the elastomeric polymer to withstand large deformations without failure made it possible to explore and implement new joint designs, for example "double-taper dovetail joints," that cannot be used with hard materials. This approach simplifies the fabrication of soft structures comprised of materials with different physical properties (e.g., stiffness, electrical conductivity, optical transparency), and provides the methods required to "program" the response of these structures to mechanical (e.g., pneumatic pressurization) and other physical (e.g., electrical) stimuli. The flexibility and modularity of this approach was demonstrated in a set of soft structures that expanded or buckled into distinct, predictable shapes when inflated or deflated. These structures combined easily to form extended systems with motions dependent on the configurations of the selected components, and, when fabricated with electrically conductive tiles, electronic circuits with pneumatically-active elements. This approach to the fabrication of hollow, 3D structures provides routes to new soft actuators.

\section{Introduction}

Soft, inflatable structures are the active elements responsible for providing the important motion (locomotion $^{[1]}$ and manipulation ${ }^{[2-5]}$ ) to soft machines and robots. ${ }^{[1,4,6,7]}$ These soft devices require combinations of materials and structures that are sometimes difficult or impossible to fabricate using techniques such as soft lithographic molding. ${ }^{[8]}$ This technique (or, rather, combination of techniques) is useful for fabricating planar structures with homogeneous 
composition, but less efficient at generating hollow, three-dimensional (3D) structures, especially when they comprise multiple materials with different mechanical or physical properties. To fabricate 3D structures that incorporate elastomeric, inflatable components for use in the actuation of soft robots and machines, we are developing methods that are based on the assembly of prefabricated, standardized pieces - thin, two-dimensional (2D) elastomeric tiles. Soft structures or devices fabricated using this approach can be "programmed," by choosing tiles made of different materials, to undergo desired changes in shape on either pneumatic expansion or contraction. These structures can provide the appropriate types and ranges of motion useful in soft machines.

In the demonstrations described in this paper, we fabricated elastomeric tiles of different compositions, using soft lithography, ${ }^{[8]}$ and joined them to form hollow, 3D structures. When the interiors of these hollow structures were pressurized with gas, they expanded and changed shape; when evacuated they collapsed (via buckling) into quite different shapes. The patterns and choices of materials used in the tiles, the arrangement of the tiles in the assemblies, and the applied pressure are the critical parameters that dictate the shape and size of the inflated (or evacuated) structures. Different structures can be generated by the inflation of tile-based assemblies that are geometrically indistinguishable in their uninflated states.

This methodology required the development of a methodology which we call "soft" joinery to connect the tiles with leak-free bonds that were sufficiently strong that they did not rupture at the pressures used (plus or minus $\sim 100 \mathrm{kPa}$ ). Soft joinery extends the strategies of joinery ${ }^{[9]}$ used for the assembly hard materials to the assembly of elastomeric tiles. Soft joinery works to connect elastomeric tiles using both dovetail joints that are common to hard materials, and a related but new structure - "double-taper dovetail joints"- that cannot be used with hard 
materials because these joints require elastomeric deformation of the tile during assembly. Using soft joinery and elastomeric tiles, we demonstrated a set of soft, shape-changing structures that may be useful for fabrication of soft machines and robots. Our approach enables the combination of elastomeric and hard polymers into structures with mechanical properties and functions defined by the designs of the structures, and the choices of the materials.

Shape-change is a strategy used in biological and synthetic systems to achieve a variety of functions, including actuation. Puffer fish inflate their bodies with water to create an intimidating silhouette ${ }^{[10]}$ to ward off would-be predators, and amoeboids utilize pseudopodialiterally "false feet," temporary cytoplasm-filled projections of the cell wall—to move through their environment and capture prey. ${ }^{[11]}$ In microrobotics, ${ }^{[12]}$ actuation may be achieved using materials that change shape upon stimulation. For example, shape-memory alloys (SMAs), ${ }^{[13]}$ following mechanical deformation, recover their original shape when exposed to heat, and piezoelectric crystals expand (or contract) along specific axes when exposed to electric fields. ${ }^{[12,14]}$ In macroscale machines/robots, shape-change actuation is demonstrated by the McKibben "air muscle,"[15] which is driven pneumatically, and iRobot's "blob-bot," which operates by stiffening portions of an inflatable soft skin using jamming. ${ }^{[16]}$ The reversible jamming of granular media was also utilized in robotics by universal grippers ${ }^{[17]}$ - the locomotion of blob-bot takes advantage of the technology developed for these devices.

The programmed shape-change of solids enables precise control of the geometrical changes a material undergoes in response to an applied stress. ${ }^{[18-23]}$ This control is achieved by designing structures with mechanical instabilities that buckle in a predictable way when subjected to mechanical deformation (e.g., compression). ${ }^{[18-20,23]}$ Following this approach it is possible to produce structures that undergo large $(>50 \%)$, isotropic volume changes, and that have negative 
Poisson's ratios. ${ }^{[19,20]}$ Shape-changing solids offer functionality that is useful to a range of applications including encapsulation, impact absorption, filtration, and rapid construction (i.e., foldable/deployable structures) ${ }^{[19]}$

We have developed a new class of soft actuators-PDMS structures containing embedded pneumatic networks (pneu-nets) - that operate by the pressurization and inflation of these pneunets. ${ }^{[4]}$ By designing systems that incorporate multiple, independently addressable pneu-nets, we have created soft machines and robots that are inexpensive, light weight, and simple to fabricate, design, and operate..$^{[1,4-7]}$

In our previous demonstrations of pneu-net-based soft machines/robots, we relied exclusively on soft lithography for fabrication. In soft lithography a master is created (for soft machines we have usually fabricated the masters using 3D printing ${ }^{[24,25]}$ ) and the topological features of the master are inversely replicated by casting and curing soft, elastomeric polymers inside them. While this approach has been successful at generating various functional devices based on the layer-by-layer assembly of roughly planar components with homogeneous material composition, soft lithography alone is limited in its ability to fabricate $3 \mathrm{D}$, hollow structures with complex topography, and especially with large, local variations in the properties of the materials.

Many strategies developed for connecting hard, planar components to form complex 3D objects have no analogs in soft materials. Fasteners (e.g., nails, screws, rivets, and nuts/bolts) are most commonly employed with hard materials, but are useful with soft materials only in restricted applications. Welding is another important method for joining hard materials, especially metals, but it works with materials that undergo thermal phase-transitions between liquid and solid states without degradation — this requirement constrains welding, when used with polymers, to a small subset (e.g., thermoplastic elastomers). Adhesives (or glues), which 
bond materials together using chemical/physical interactions, offer versatility in connecting components because they work with many types of materials (e.g., wood, fabric, metal, polymers, etc.), and they are simple to apply without specialized tools. Glues, however, have the limitation that their bonding strength is proportional to the surface area of contact between the bonded components, and to the interfacial free energies of the various interfaces between the bonded surfaces, and they must be often used in conjunction with other methods of connection such as fasteners or joinery.

Joinery is derived from the craft of woodworking and includes methods for combining different wooden elements that use only the material itself and a binder (e.g., glue). ${ }^{[9]}$ In joinery, the strategy is to design overlaps_-joints—-between components that, using a "lock-and-key" relationship, resist separation of the components (in one or more directions) while increasing the surface area of contact available for gluing. Some popular examples of joints include: (i) butt joints, (ii) dado joints, and (iii) lap joints. Dovetail joints (belonging to the lap joint family) are widely used because of their large area of contact and their ability to withstand tensile load. The basic dovetail joint (Figure 1A), consists of "pins" that interlock with a corresponding set of "tails" in the mating piece. Other varieties include through, half-blind, secret, and sliding dovetail joints. ${ }^{[9]}$

\section{Design and Fabrication of Inflatable Structures}

\subsection{Elastomeric Tiles for the Fabrication of Inflatable Structures}

We expand the methods available for fabricating three-dimensional soft structures by combining soft lithography ${ }^{[8]}$ with the concept of tiling (which we define as the fabrication of structures based on the assembly of tiles). Our strategy was to create a set of elastomeric tiles-thin, geometrically regular, 2D sheets of elastomeric polymers - that could be easily fabricated using 
Figure 1. (A) Schematic of a single-taper dovetail (top) and the mating pins (bottom). (B) Schematic of a double-taper dovetail (top) and the mating pins (bottom). (C) Top down view of the tiles used to assemble a hollow, elastomeric cube. Opposing faces of the cube had the same color; all faces were fabricated in PDMS. This design utilizes a single-taper joint and requires three distinct types of elastomeric tiles to assemble the cube. (D) Optimal sequence of assembly (other sequences are possible). The orientation of one face is marked with an arrow. Coating the surface of each edge with liquid PDMS prepolymer followed by curing of the assembled joint created an airtight seal. No prepolymer was used in this illustration. (E) A fully assembled PDMS cube. The colored PDMS helps visualize the interlocking dovetail joints. 
Figure 1.

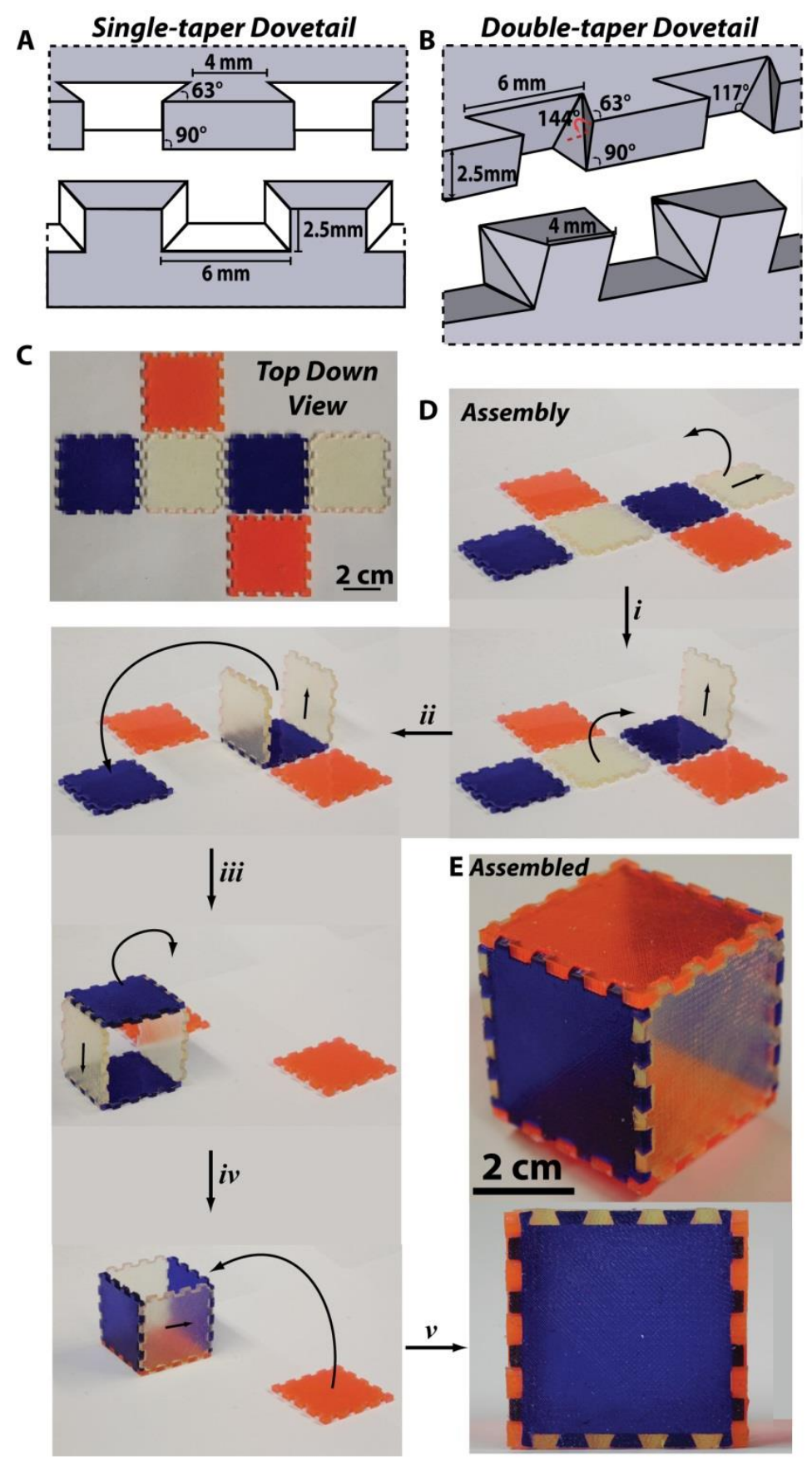


soft lithography, and combined to form 3D structures with thin walls and complex mechanical responses in shape (e.g., to inflation or deflation).

\subsection{Soft Joinery}

Our strategy required the connection of thin, elastomeric pieces at edges that overlap by only a few millimeters. We developed soft joinery to overcome the challenges associated with fabrication using elastomeric tiles: First, the edges of the tiles have little surface area to enable strong bonding. Second, the flexibility of the tiles makes it difficult to maintain the appropriate alignment between them during bonding of simple joints (e.g., butt joints). Finally, for the fabrication of pneumatic actuators, the interface between tiles must withstand the high tensile strains associated with pneumatic pressurization.

We have overcome these challenges using soft joints based on the dovetail design. The soft dovetail joints increased the surface area available for gluing the thin ( $\sim 3 \mathrm{~mm}$ in thickness) tiles together, and mechanically reinforced the interface between the tiles to withstand tensile force associated with pneumatic actuation. The dovetail joints also have the advantage that they provide some measure of encoding to the fabrication process - the joints consist of complimentary pin and tail edges (Figure 1A, B; Figure S1 and Figure S2), which simplify their alignment during the assembly of the tiles and control if two tiles fit together. In the case of a cube, the dovetail joints require (at minimum) two types of tiles with unique edge designs (Figure 1A, B, C-E; Figure S1 and Figure S2). Although we focused on the fabrication of hollow elastomeric cubes, obvious extensions of this method lead to other geometrical shapes, such as tetrahedrons, octahedrons, and other faceted shapes.

\subsection{Design of Soft Joints}


Soft materials have mechanical properties that make it possible to explore joint designs that are incompatible with hard materials. We designed dovetails that taper in a single direction or in two orthogonal directions (Figure 1A, B; Figure S1 and Figure S2) to form interlocked joints between the elastomeric tiles. We used taper angles that were more acute $\left(<65^{\circ}\right.$, Figure $\left.1 \mathrm{~A}, \mathrm{~B}\right)$ than those found in dovetails designed for hard materials (e.g., wood or structural plastics, where angles of $70^{\circ}$ to $80^{\circ}$ are common) because this design increases the surface area of the joint and the extent to which the pieces interlock. Dovetail joint designs with acute-taper angles are rarely used in hard (and potentially brittle) materials because they lead to fracture at the tips or base of the tail (where material is thinnest), and their susceptibility to mechanical failure often offsets, if not outweighs, the potential improvement in strength gained from the increase in surface area of the joint and the amount of interlock. ${ }^{[9]}$ In contrast, the elastomeric polymers polydimethylsiloxanes (PDMS, Sylgard ${ }^{\circledR} 184$ ) and Ecoflex ${ }^{\circledR} 0030$ that we used to fabricate our tiles can tolerate much larger deformations (160\% and 900\% elongation at break for PDMS and Ecoflex ${ }^{\circledR} 0030$ respectively) on stretching or bending without material failure, and we can realize the advantages of more acute taper angles in the designs of the joints we use.

In addition to dovetails with acute taper angles, it was also possible to implement dovetail designs that taper in two directions, double-taper dovetails (Figure 1B; Figure S2), and these joints resist tensile strain in orthogonal directions. This joint is inapplicable to connecting solid pieces of rigid hard material, like wood, because two hard mating pieces cannot be deformed to interlock without damage. In contrast, the flexibility and elasticity of soft materials allowed us to assemble elastomeric tiles with the double-taper dovetail joints by squeezing the pins and tails together. The double-taper design can be used to join elastomeric tiles to other elastomeric tiles 
or to hard tiles (e.g., those made of rigid thermoplastics), but it cannot be used to join hard tiles to other hard tiles.

\subsection{Pneumatic Actuation of Soft Structures Assembled from Tiles}

We coated the soft dovetail joints with liquid PDMS pre-polymer glue, assembled them to form hollow elastomeric cubes, and thermally cured the assembly to form structures with air-tight edges. We then used compressed air or vacuum to actuate the structures pneumatically because: (i) gas is nearly inviscid, and allows rapid inflation and pressurization, (ii) air is readily available in the atmosphere, (iii) there is no significant change in mass after the structures are inflated, and (iv) a single connection — one pneumatic line — can inflate and deflate the same structure. We have previously demonstrated the usefulness of pneumatics in the actuation of a variety of soft machines. ${ }^{[1,4-7]}$

The choice of which joint design to use in these structures depends on the intended positive operating pressure of the device and the material used to fabricate the tiles. We demonstrated a set of structures that were fabricated from combinations of PDMS and Ecoflex ${ }^{\circledR}$ tiles that we operated at equilibrium pressures at or below $\sim 115 \mathrm{kPa}$. Under these conditions both the singletaper and double-taper joint designs are functional. At higher pressures $(\sim 120 \mathrm{kPa})$ we observed material failure before joint failure (Figure S5). When we assembled structures from only PDMS (the stiffer of the two elastomers investigated) tiles and inflated them to failure, the point where the device ruptured, and the corresponding pressure depended on whether single-taper or double-taper joints were used-single-taper devices failed at the joints at lower pressures while double-taper devices failed at the joints at higher pressures (Figure S5). We focused on several low-pressure demonstrations of shape-changing devices assembled using single-taper joints because structures based on these joints are easier to assemble (in large numbers) by hand than 
those based on double-taper joint designs while being functionally equivalent; however, for devices based on stiffer elastomeric materials intended for higher pressure applications the double-taper joint design would be more appropriate.

\section{Results and Discussion}

\subsection{Shape Change via Controlled Expansion}

We created a variety of soft, inflatable cubes from elastomeric tiles using soft joinery. Upon the application of positive pressure to the hollow interior of the cubes, each structure expanded into a unique shape that depended upon the extensibility of the tiles used for each of the different faces of the cube (Figure 2, Video S1). This ability to program - by using tiles with different mechanical properties and by modifying their positions in the assembly - the inflation of hollow 3D structures into topographically complex geometries is an advantage of our approach and the pneumatic actuation of these structures may find use in soft machines. The reversible shape change demonstrated by these soft structures has no analog in the science of hard materials.

Using tiles fabricated from either PDMS or Ecoflex ${ }^{\circledR}$ 0030, we assembled 7 out of the 15 possible cube structures that can result from a set of tiles consisting of two unique types (Figure 2 and Figure S6). To illustrate the ability of these soft structures to do work, we inflated a cube (to an equilibrium pressure of $\sim 115 \mathrm{kPa}$ rapidly using a positive pressure of $100 \mathrm{kPa}$ above atmospheric pressure) made of six Ecoflex ${ }^{\circledR}$ tiles lifting a $3 \mathrm{~kg}$ load that was placed on top of it (Figure S7). The joints did not fail. In a separate test of an equivalent device we inflated the structure to $\sim 120 \mathrm{kPa}$ (with no load) at which point the Ecoflex ${ }^{\circledR}$ material itself failed while the soft joints remained intact (Figure S5) — the joints are not a limitation to pressure, the material is.

We achieved anisotropic shape-change in cubes assembled from tiles patterned with two elastomers of different stiffness. In one example we fabricated cubes from tiles with extensible 
Figure 2. (A-C) Shape-changing, cubic structures fabricated from tiles of varying extensibilities. Each panel includes a schematic that illustrates the material used for each tile (PDMS is hatched and Ecoflex ${ }^{\circledR}$ is white). In each panel the top photographs show the actuators under isobarometric $(\Delta \mathrm{P}=0)$ pressure and the bottom show the actuator under positive $(+\Delta \mathrm{P})$. For scale, the non-deformed edges of the cubes are all $4.5 \mathrm{~cm}$. A positive pressure of $+\Delta \mathrm{P} \sim 10$ kPa was used. 
Figure 2.

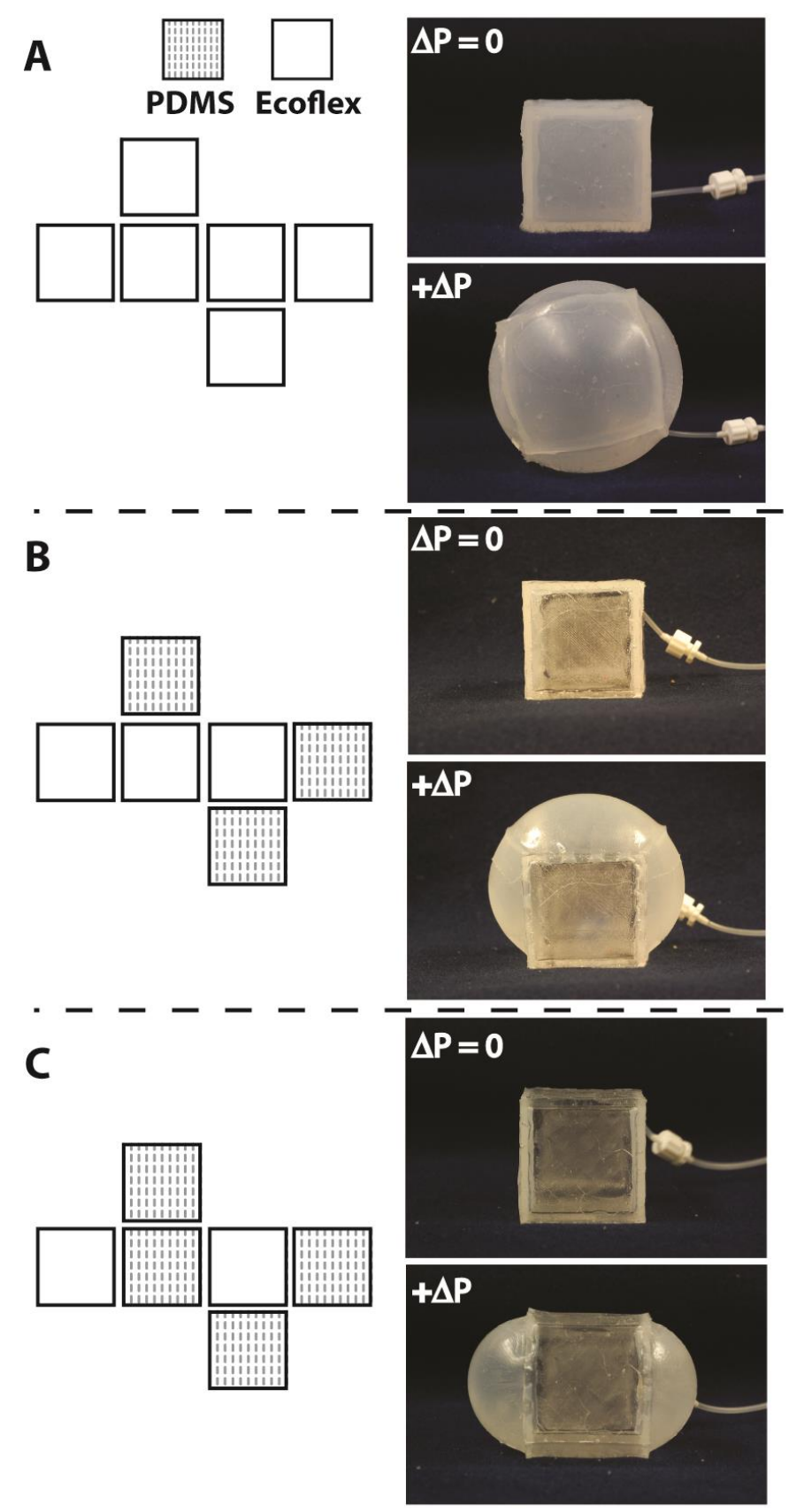


elastomer at the center (or strips of extensible elastomer that cross at the center) and less extensible elastomer in the surrounding area (Figure 3A, B, D, and E). Actuation with positive pressure led to pronounced, anisotropic expansion at the centers of the individual faces of the cubes (Figure 3C, F and Video S2). This result highlights another level of sophistication made possible following our approach. Increasing the variety of materials used for fabrication of the tiles will significantly expand our ability to program the properties of these structures.

\subsection{Shape Change via Controlled Buckling}

We also examined how the material composition of the elastomeric tiles and the arrangement of these tiles in soft, cubic structures influenced their collapsed geometry. Collapse of the structures was achieved by the application of negative pressure (approximately $65 \mathrm{kPa}$, or $-\Delta \mathrm{P}=35 \mathrm{kPa}$ ) to the interior of the structures; the resulting forces from the atmosphere caused the tiles that formed the walls to buckle. The application of vacuum to the interior of cubic structures fabricated by combining six tiles of homogenous material composition resulted in the formation of collapsed structures with ill-defined geometries (Figure 4A, G, M; and Figure S8).

In contrast, cubes fabricated with tiles that included rationally patterned regions of extensible and relatively inextensible elastomers collapsed into shapes with distinct geometries that were different from one another and from the initial cubic state (Figure 4B-F, H-L, and N-R and Video S3). Successful designs incorporated Ecoflex ${ }^{\circledR}$ at the edges or the centers of the tiles that, when assembled, yielded cubes with corners and/or faces that were softer than PDMS, which made up the remainder of the structures. Upon the application of vacuum, the soft faces/corners would flex inward first "pulling" on the stiffer PDMS regions of the structure, inducing buckling. We have illustrated the simplest possible designs using only two types of elastomeric polymers; there 
Figure 3. Inflatable cubes fabricated with tiles comprised of more than one material. (A, D) Schematic showing the tiles used to fabricate the cube. The PDMS regions of the tile are marked with hatch marks and the Ecoflex ${ }^{\circledR}$ regions are solid white. (B, E) Photographs of the actuator with no pressure applied. $(\mathrm{C}, \mathrm{F})$ Shape change achieved using positive pressure $(+\Delta \mathrm{P} \sim 10 \mathrm{kPa})$. 
Figure 3.
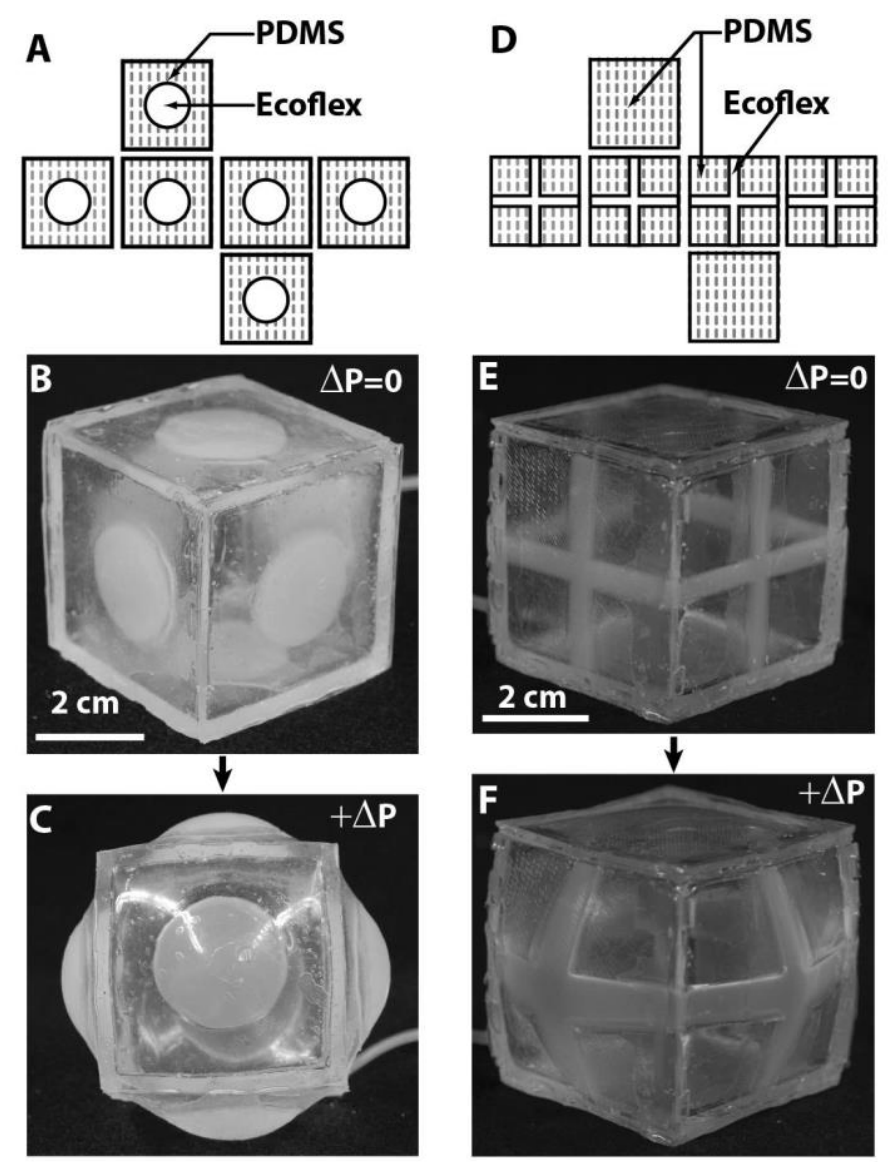
Figure 4. Collapse of cubes fabricated with tiles comprised of a single material and more than one material. (A-F) Schematic showing the tiles used to fabricate the cubes. The PDMS regions of the tile are marked with hatch marks and the Ecoflex ${ }^{\circledR}$ regions are solid white. (G-L)

Photographs of the actuator with no pressure applied. (M-R) Photographs of buckled cubes after negative pressure $(-\Delta \mathrm{P} \sim 35 \mathrm{kPa})$ was applied. 
Figure 4.
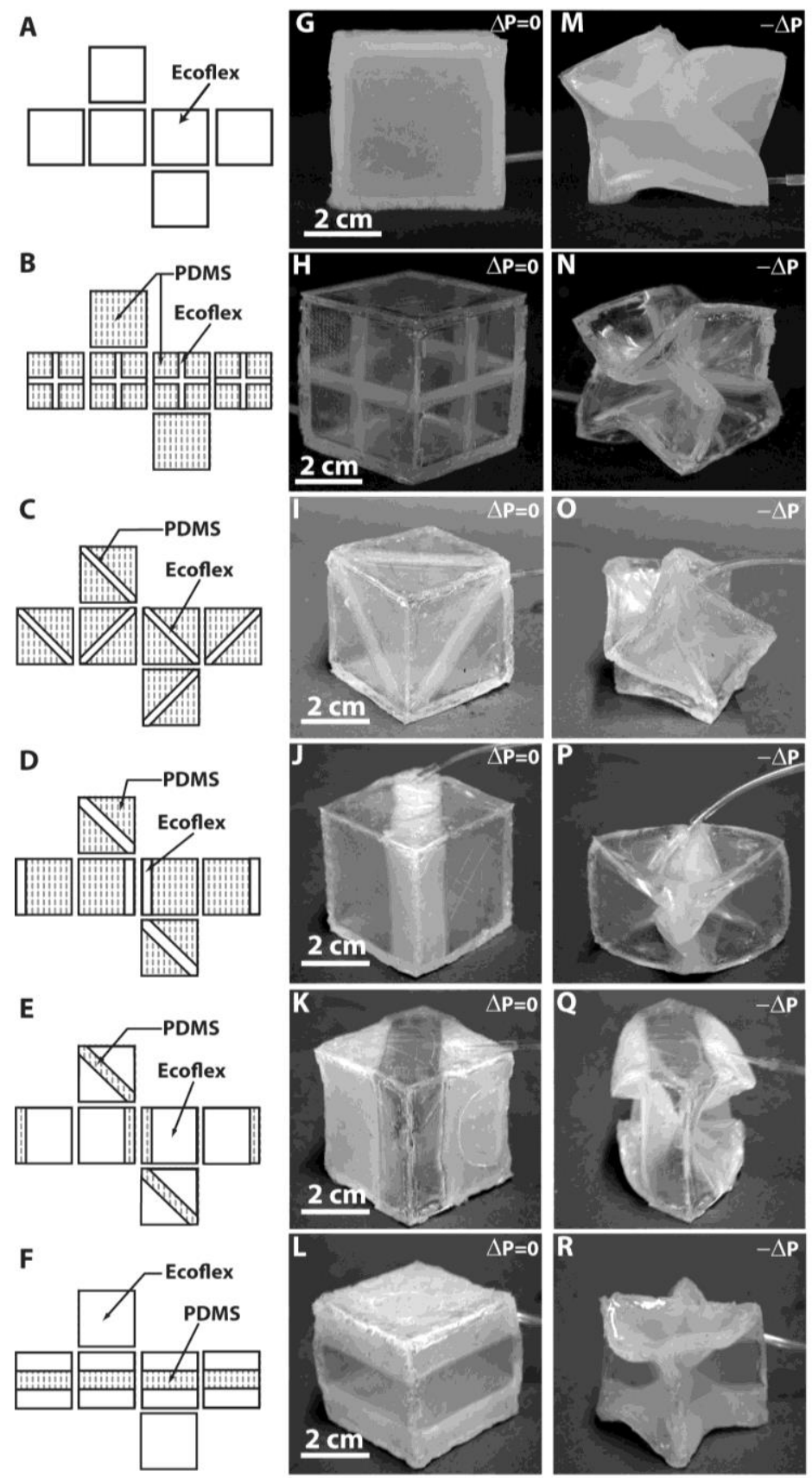
are many more possibilities that could use more intricate patterns of a larger variety of materials, and produce more complex buckling behaviors.

\subsection{Extended Structures from Assemblies of Cubes}

Utilizing a simple "peg/recess" connector that we have demonstrated previously ${ }^{[26]}$ (Figure S3, S4, and Supporting Information text), it is possible to connect the inflatable cubes together to form extended structures with independently addressable segments (Figure 5). To illustrate this concept we assembled a set of cubes into two different linear configurations (Figure 5C-D). In each example the cubes were held together at the interface between two neighboring PDMS faces using a complimentary peg/recess connector, which enables the reversible assembly of these cubes into a variety of different configurations. In one example, we joined two cubes "back-to-back" (Figure 5A) and inflated them simultaneously lifting the assembly upwards against gravity (Figure 5B, C). In another demonstration, we assembled three cubes to form a tower and deflated each segment sequentially to compress the tower to $50 \%$ of its original height (Figure 5D). We then connected each segment to a source of pressurized air and re-inflated the individual cubes restoring the tower to its original height (Figure 5D). By inflating the segments beyond their original volume we expanded the height of tower by 10\% (Figure 5D and Video S5). Beyond collective one dimensional motion such as expansion and contraction, a properly configured assembly of cubes can also generate 2D motion such as bending (Figure 5E). In these demonstrations, we focused on the simplest configurations and motions, but by extending this methodology to include a larger number of inflatable structures, connectors that position the structures in different architectures (e.g., a "T" connector), and structures of different geometries (e.g., prisms or other polyhedra), it will be possible to construct soft machines for specific functions. 
Figure 5. (A) Annotated photograph showing the peg/recess press connectors used to join two cubes (of the type shown in Figure S6A) and the Luer-Lok connectors used for the pneumatic lines. (B) Symbols used to show the pressure state of the individual actuators in panels C-E. (C) Photograph showing the two cubes from $\mathrm{A}$ assembled and inflated using positive pressure $(+\Delta \mathrm{P}$ $\sim 10 \mathrm{kPa}$ ). (D) A tower constructed from three cubes (of the type shown in Figure S6B).

Progressing from left to right, the tower is collapsed using negative pressure $(-\Delta \mathrm{P} \sim 35 \mathrm{kPa})$ and expanded back to full size using positive pressure $(+\Delta \mathrm{P} \sim 10 \mathrm{kPa})$. The tower was anchored to a glass base. (E) Photograph of a line of three cubes (of the type shown in Figure S6A) whose bases were fixed to a strip of PDMS using the peg/recess connector. Progressing from left to right, the assembly is bent into an arch using positive pressure and contracted back into a row using negative pressure. 
Figure 5.
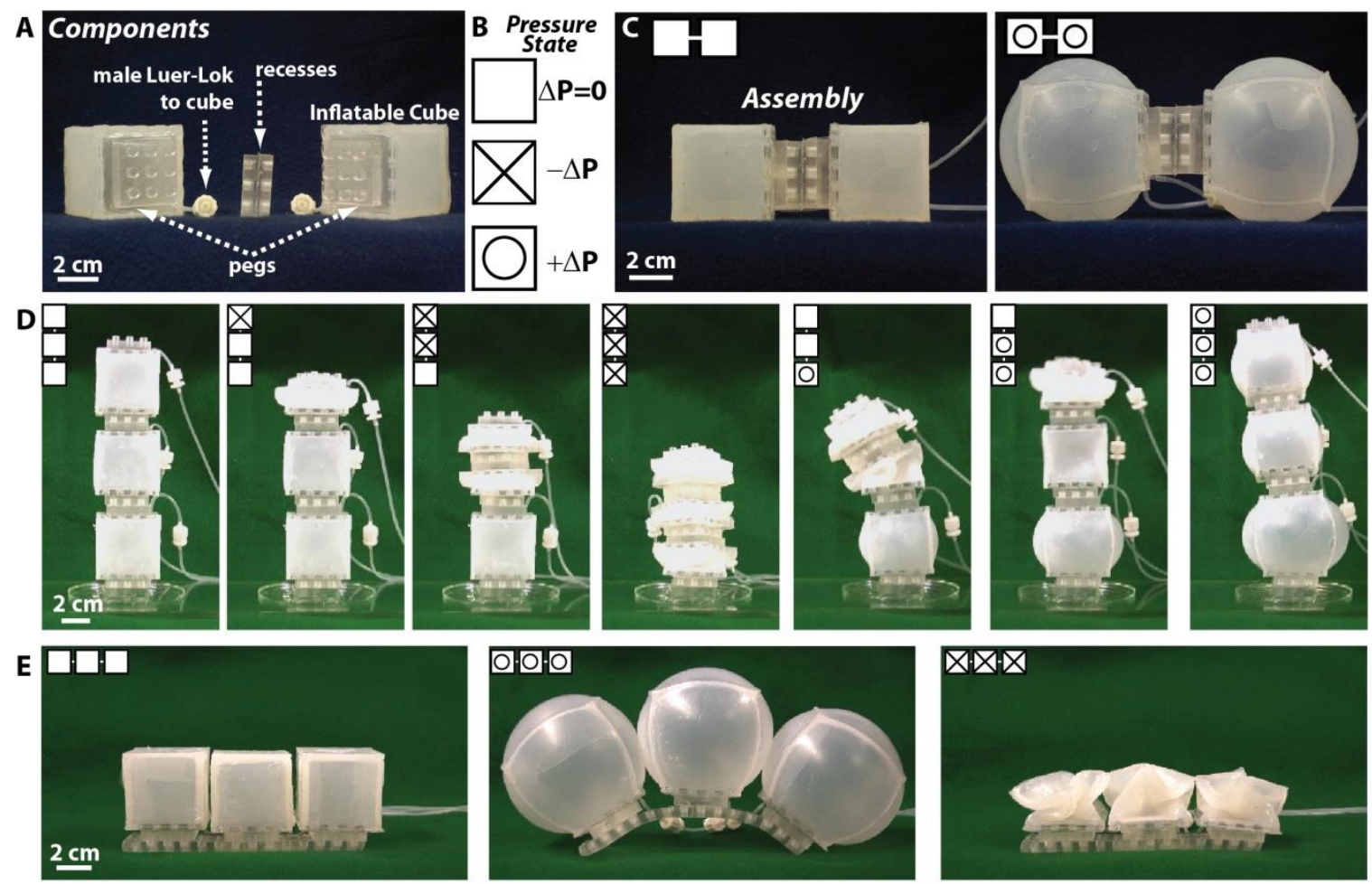


\subsection{Heterogeneous Materials for Multi-functional Structures}

We have focused on controlling the mechanical properties of elastomeric tiles, and through them the mechanical properties of the final assembly. A strategy based on the assembly of tiles can, however, be extended to other properties (e.g., electrical conductivity, optical transparency, or magnetic responsiveness). Using this extension, it is possible to create multi-functional structures with characteristics that depend on the properties of the elastomeric tiles used in their assembly. To demonstrate this concept, we fabricated tiles with conductive regions and assembled inflatable cubes from them (Figure 6). The conductive "patches" of the tiles were composed of silicone elastomer containing embedded metallic wool that supported conductivity, but that did not significantly alter the mechanical properties of the elastomer itself (as evidenced by the observed isotropic inflation). We connected a light-emitting diode (LED) to the conductive patches inside of the cube as a means to visualize the formation of a conductive pathway through the elastomeric tiles and across the cubes themselves. When this structure is placed between two metallic plates and inflated, the conductive patches on opposing faces of the cube made electrical contact with the plates, which were held at a $3 \mathrm{~V}$ bias, and a completed circuit was formed. If the orientation of the structure relative to the polarity of the plate electrodes was correct, the LED was biased in the forward direction allowing current to flow through the circuit. This current powered up the LED illuminating the inflated structure (Figure 6A-D). Rotating the structure about an axis parallel to the surface of the plate electrodes broke electrical contact with the conductive patches and the LED switched off (Video S6); likewise, flipping the orientation of a structure in an illuminated state upside down, led to a negative bias across the LED and no light is emitted (Figure 6E and Video S6). Rotating the inflated structure, as connected in Figure 6C, along the axis passing through the pair of conductive patches in 
Figure 6. Inflatable structure fabricated from tiles with conductive patches bridged by an internal LED. (A) Photograph of the uninflated cube between two oppositely charged metal plates. The dark portions at the center of each tile are conductive. In the interior of the cube is an LED whose anode was connected to three faces of the cube and whose cathode was connected to the remaining three faces (all connections were made to the conductive patches). (B) Schematic showing the experimental setup (left) and the circuit diagram (right) corresponding to the crosssection indicated in the schematic. (C, D) Photograph of an inflated cube that was illuminated as a result of the anode (face 1) and cathode (face 2) of the LED making contact with the charged plates in the forward bias direction. (E) Photograph and schematic sequence showing the inflated cube as it was rolled between the plates from the forward bias configuration, $\mathrm{C}$, to the reverse bias configurations, $i$ and $i i$, to a second forward bias configuration, $i i i$, and back to the original forward bias configuration in C. (F) Photograph and schematic sequence showing an inflated cube being rotated $360^{\circ}$ between the plates while maintaining electrical contact. Note the small tube seen in these images was used to inflate (A-F) and rotate $(\mathrm{F})$ the cube. 
Figure 6.
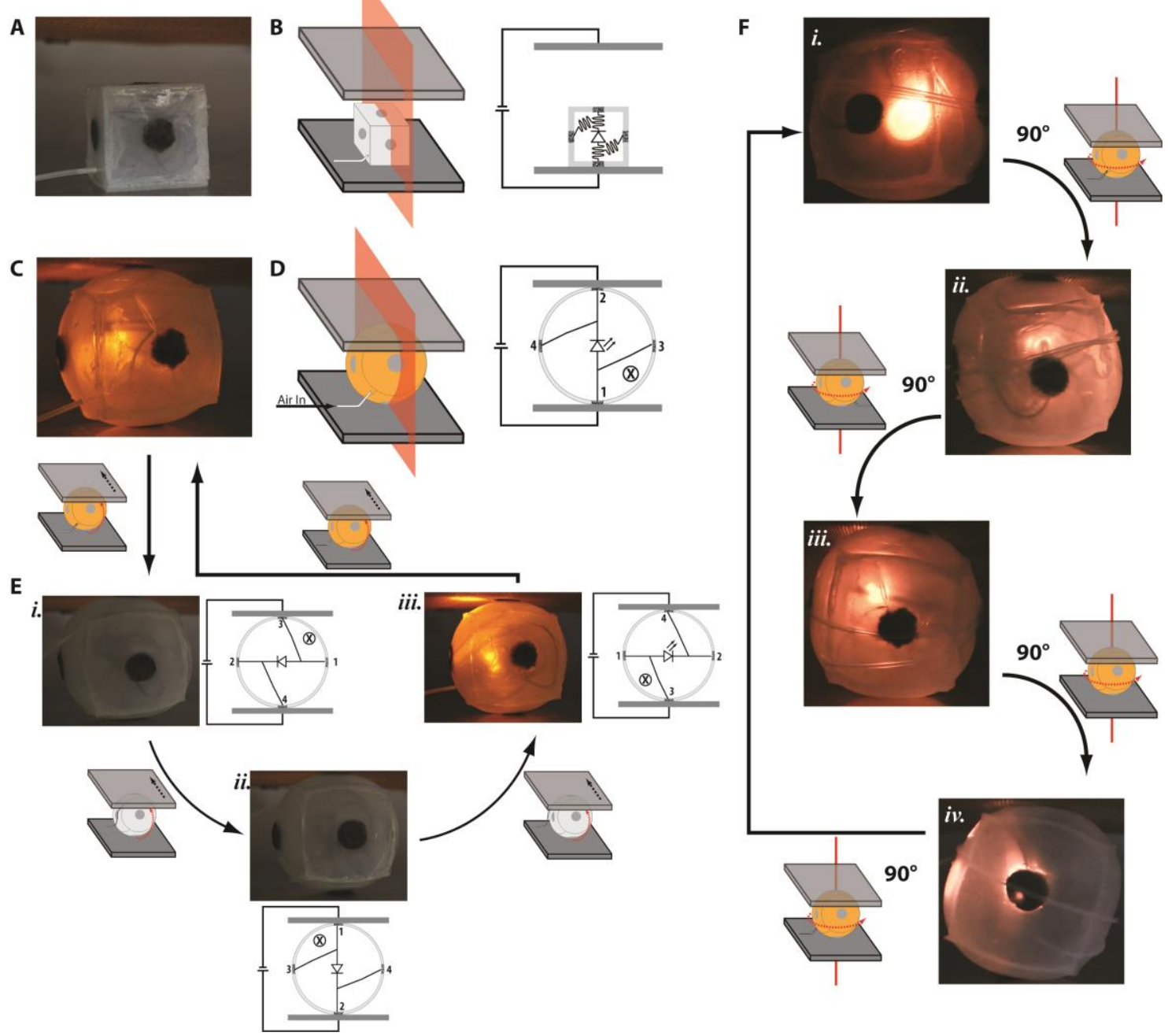
contact with the electrodes, maintained the positive bias across the LED and the structure remained illuminated (Figure 6F). The dependence of the on/off state of the LED on the direction of bias provided a method to encode (based upon the configuration of the electrical contacts inside the structure) information about the orientation of a structure that was geometrically symmetric.

\section{Conclusions}

Soft joinery of elastomeric tiles provides a practical method to fabricate a class of soft, shapechanging devices based on inflatable, hollow, 3D structures. We believe that these structures are useful prototypes to components of soft machines/robots. Using this approach, the geometrical complexity and the variety of shapes that we can produce, and the number of different properties (mechanical, electrical, optical) that we can incorporate into the final structures, is large, and would be difficult to replicate with other techniques. When assembled into extended structures, the types of cubes used, their spatial arrangement, and their sequential inflation/deflation provides a range of motions and properties beyond what is possible with a single element. These extended structures can be assembled, disassembled, and reassembled into different configurations; this ease of assembly (both reversible and irreversible) enables the fabrication, design, and testing of soft machines and robots new 3D architectures that would otherwise be hard to fabricate.

Soft joinery enables the connection and bonding of soft components (tiles in this case) to other soft components, and of soft components to hard components, and makes it possible to form one monolithic piece from the component tiles - a capability that is useful for the fabrication of hybrid hard/soft structures, ${ }^{[7]}$ and not easily achieved using other methods. Certain soft joint designs, such as double-tapered dovetails, are unique to elastomeric tiles, and not 
applicable to the connection of hard materials to other hard materials (because they could not be assembled); these double-tapered dovetails have no precedent in joinery. ${ }^{[9]}$ By expanding the range of materials used in joinery to include soft materials, we can now explore new joint designs (for example, the double tapered dovetails) and we anticipate that fields concerned with hybrid structure/actuator design, such as mechanical engineering and robotics, will benefit from this new capability. We focused on soft, silicone-based elastomeric polymers in this demonstration, but our strategy can be extended to stiffer elastomers (e.g., particle- or fibertoughened rubber composites ${ }^{[27]}$ ) and to the connection of these materials to hard materials (e.g., sheet steel or rigid thermoplastics).

Using this approach, it is possible to incorporate additional functionality, such as electrical conductivity, into the final structures. The large amount of void space inside the hollow structures opens the opportunity to embed electronics, batteries, and compressors inside hollow, elastomeric actuators. Our initial demonstration of this concept used an LED as illustrationextensions of this idea would produce more advanced systems. Such co-localization of actuators, power sources, and control systems is not easily achievable using other structures, and will lead to new designs for soft machines/robots. We derived the designs of the structures that we fabricated and tested empirically, but they are conceptually related to shape-changing solids. [18-20,23] Future demonstrations will apply the design rules established in these related structures to optimize and expand the varieties of motions achievable by soft structures. Further, the advantages this approach offers to the fabrication of topologically complex (in terms of mechanical and physical properties) structures will provide routes to new shape-changing structures with additional functionalities (e.g., electrical conductivity) that were previously inaccessible. 


\section{Experimental}

Please see the Supporting Information.

\section{Supporting Information}

Supporting Information is available from the Wiley Online Library or from the author.

\section{Acknowledgments}

The design and fabrication of soft joints and inflatable structures based on them was supported by the Department of Energy (DE-FG02-00ER45852.), which also funded S.A.M, S.W.K, and

J.L. The development of aspects related to soft machines was supported by the Defense Advanced Research Planning Agency (W911NF-11-1-0094), which also supported R.F.S. and A.A.S. J.T. was supported by the Harvard Summer School Proctor Program and the Harvard College Research Program. We thank the Materials Research Science and Engineering Center at Harvard University (supported by the National Science Foundation under award number DMR0820484) for access to facilities.

\section{References}

[1] R. F. Shepherd, F. Ilievski, W. Choi, S. A. Morin, A. A. Stokes, A. D. Mazzeo, X. Chen, M. Wang, G. M. Whitesides, P. Natl. Acad. Sci. U.S.A. 2011, 108, 20400.

[2] K. Suzumori, S. IIkura, H. Tanaka, in Proceedings IEEE Micro Electro Mechanical Systems, IEEE Press, Piscataway, NJ, USA 1991, 204.

[3] K. Suzumori, Robot Auton. Syst. 1996, 18, 135.

[4] F. Ilievski, A. D. Mazzeo, R. E. Shepherd, X. Chen, G. M. Whitesides, Angew. Chem. Int. Edit. 2011, 50, 1890.

[5] R. V. Martinez, J. L. Branch, C. R. Fish, L. H. Jin, R. F. Shepherd, R. M. D. Nunes, Z. G. Suo, G. M. Whitesides, Adv. Mater. 2013, 25, 205. 
[6] S. A. Morin, R. F. Shepherd, S. W. Kwok, A. A. Stokes, A. Nemiroski, G. M. Whitesides, Science 2012, 337, 828.

[7] S. W. Kwok, S. A. Morin, B. Mosadegh, J.-H. So, R. F. Shepherd, R. V. Martinez, B.

Smith, F. C. Simeone, A. A. Stokes, G. M. Whitesides, Adv. Funct. Mater. 2013, 24, 2180.

[8] Y. N. Xia, G. M. Whitesides, Annu. Rev. Mater. Sci. 1998, 28, 153.

[9] G. Ellis, Modern Practical Joinery, B.T. Bratsford, London, UK 1908.

[10] E. L. Brainerd, J. Morphol. 1994, 220, 243.

[11] P. J. M. Van Haastert, P. N. Devreotes, Nat. Rev. Mol. Cell Biol. 2004, 5, 626.

[12] K. Y. Ma, P. Chirarattananon, S. B. Fuller, R. J. Wood, Science 2013, 340, 603.

[13] M. Sreekumar, T. Nagarajan, M. Singaperumal, M. Zoppi, R. Molfino, Ind. Robot 2007, $34,285$.

[14] H. Irschik, Eng. Struct. 2002, 24, 5.

[15] H. F. Schulte Jr., The Characteristics of the McKibben Artificial Muscle, Wachington, DC, USA 1961.

[16] E. Steltz, A. Mozeika, N. Rodenberg, H. M. Jaeger, in Proceedings of the 2009 IEEE/RSJ International Conference on Intelligent Robots and Systems, IEEE Press, Piscataway, NJ, USA 2009, 5672.

[17] E. Brown, N. Rodenberg, J. Amend, A. Mozeika, E. Steltz, M. R. Zakin, H. Lipson, H. M. Jaeger, P. Natl. Acad. Sci. U.S.A. 2010, 107, 18809.

[18] S. Alben, M. P. Brenner, Phys. Rev. E 2007, 75, 056113.

[19] J. Shim, C. Perdigou, E. R. Chen, K. Bertoldi, P. M. Reis, P. Natl. Acad. Sci. U.S.A. 2012, 109, 5978. 
[20] S. Babaee, J. Shim, J. C. Weaver, E. R. Chen, N. Patel, K. Bertoldi, Adv. Mater. 2013, 25, 5044.

[21] L. J. Burton, N. Cheng, C. Vega, J. Andres, J. W. M. Bush, Bioinspir. Biomim. 2013, 8, 044003.

[22] Z. Y. Wei, Z. V. Guo, L. Dudte, H. Y. Liang, L. Mahadevan, Phys. Rev. Lett. 2013, 110, 215501.

[23] E. H. Yong, D. R. Nelson, L. Mahadevan, Phys. Rev. Lett. 2013, 111, 177801.

[24] J. A. Lewis, Adv. Funct. Mater. 2006, 16, 2193.

[25] H. Lipson, M. Kurman, Fabricated: The New World of 3D Printing, John Wiley and Sons, Inc., Indianapolis, IN, USA 2013.

[26] S. A. Morin, Y. Shevchenko, S. W. Kwok, R. F. Shepherd, A. A. Stokes, J. Lessing, G. M. Whitesides, Adv. Funct. Mater. 2014, submitted.

[27] R. F. Shepherd, A. A. Stokes, R. M. D. Nunes, G. M. Whitesides, Adv. Mater. 2013, 25, 6709. 